CLINICAL REVIEW

\title{
The heritability of insomnia: Systematic review and meta-analysis of twin studies
}

\author{
Juan J. Madrid-Valero a, *, María Rubio-Aparicio a , Alice M. Gregory ${ }^{\text {b }}$, \\ Julio Sánchez-Meca ${ }^{\mathrm{c}}$, Juan R. Ordoñana ${ }^{\mathrm{d}, \mathrm{e}}$ \\ a Department of Health Psychology, Faculty of Health Science, University of Alicante, Spain \\ ${ }^{\mathrm{b}}$ Department of Psychology, Goldsmiths, University of London, London, United Kingdom \\ ${ }^{c}$ Department of Basic Psychology and Methodology, University of Murcia, Spain \\ ${ }^{\mathrm{d}}$ Department of Human Anatomy and Psychobiology, University of Murcia, Spain \\ e Murcia Institute of Biomedical Research, IMIB-Arrixaca, Spain
}

\section{A R T I C L E I N F O}

\section{Article history:}

Received 31 July 2020

Received in revised form

7 October 2020

Accepted 13 November 2020

Available online 21 January 2021

\section{Keywords:}

Dizygotic

Genetics

Heritability

Insomnia

Monozygotic

Twins

\begin{abstract}
S U M M A R Y
Twin studies have consistently found that genetic factors explain a substantial proportion of the variance for insomnia. However, studies vary widely in their heritability estimates. Therefore, this meta-analysis aimed to: 1) Estimate the mean heritability of insomnia; 2) Assess heterogeneity among twin studies of insomnia; and 3) Search and analyse characteristics of the studies (moderator variables) that may explain heterogeneity among estimates. For this purpose, separate meta-analyses were carried out for MZ and DZ correlations and for heritability estimates by assuming random-effects models. Thirteen independent samples were included in this meta-analysis. The heterogeneity index for heritability estimates was significant in both best fitting models $\left(\mathrm{I}^{2}=98.77, P<.0001\right)$ and full models $\left(\mathrm{I}^{2}=97.80, P<.0001\right) . \mathrm{MZ}$ correlations were higher $(0.37 ; 95 \% \mathrm{CI}$ : $0.31, .43)$ than $\mathrm{DZ}$ correlations $(0.15 ; 95 \% \mathrm{CI}: 0.12, .18)$. A mean heritability of 0.39 (95\%CI: $0.32, .44)$ was found for insomnia. These results highlight the role of genetic factors in explaining differences among the population on insomnia and Emphasize heterogeneity among studies. Further research is needed to identify variables that could explain this heterogeneity.
\end{abstract}

(c) 2021 Elsevier Ltd. All rights reserved.

\section{Introduction}

Insomnia is characterised by a difficulty falling asleep or staying asleep. As is the case for other sleep disorders, the aetiology of insomnia is not fully understood [1], but both genetic and environmental influences are assumed to play a role in its genesis and development. Twin studies and, more recently, analyses using genetic variants determination (e.g., polygenic risk scores) increase our understanding of the interplay between genes and the environment in insomnia, with the ultimate objective of offering clues for improving our diagnostic and treatment capability.

Abbreviations: A, additive genetic factors; C, common shared environmental factors; CBT-I, Cognitive-behavior-therapy for insomnia; D, non-additive genetic factors; DZ, dizygotic twins; DZOS, dizygotic twins opposite sex; E, non-shared environmental factors; GWAS, genome-wide association studies; MZ, monozygotic twins.

* Corresponding author. Department of Health Psychology, Faculty of Health Science, University of Alicante, 03690, Alicante, Spain.

E-mail address: juanjose.madrid@ua.es (J.J. Madrid-Valero).
Insomnia is highly prevalent and it is one of the most common complaints in medical practice [1,2]. Prevalence estimates of insomnia range from 6 to 33\% depending on the definition used [2-5]. Symptoms of insomnia occur despite adequate opportunities for sleep and can impact daytime functioning [1,6]. Insomnia has negative consequences for almost every single aspect of mental and physical health, from chronic pain to psychosis [7-16]; and its economic burden is high with an average annual per-person cost (direct and indirect combined) of $\$ 5010$ for individuals with an insomnia disorder or $\$ 1431$ for individuals presenting with symptoms, as compared to $\$ 421$ for good sleepers. The largest proportion of these expenses was attributable to work absences and reduced productivity [17]. In another study, Medicare beneficiaries with untreated insomnia had higher healthcare utilization and costs across all points of service [18]. Helpful treatments for insomnia are available. For instance, cognitive behavior therapy for insomnia (CBT-I) has proven to be effective and cost-effective $[19,20]$ and it is often recommended as the treatment of choice [1], although it is not always utilised the most [21]. 
Despite its relevance and all that is known regarding insomnia, the precise nature of its aetiology remains elusive. Twin studies have been one of the main sources of information about the relative magnitude of genetic and environmental factors on the genesis and development of insomnia. This method has proven to be a useful tool for research [22] as twin studies allow the disentanglement of genetic and environmental factors on a phenotype, trait or disorder [23] and provide estimates of heritability, which can be defined as the proportion of phenotypic variance explained by genetic factors. Heritability can be estimated using a variety of genetically informative methods, but estimation from twin studies remain relevant because of their ability to capture genetic effects as a whole, including those of rare variants and the cumulated influence of common genetic variants of tiny effect. In addition, twin studies allow for the analysis of the relative impact of shared environmental factors on a trait.

However, there is a wide variety of results; heritability estimates range from around 0.30 to 0.60 [24-27]. That is not surprising as these studies have been performed in different populations, at different times and in different locations; and heritability is a population statistic which, therefore, may vary from one population to another [28].

Heterogeneity in results can be explained in part by the approach taken to assess heritability. GWAS studies have also been used to estimate the variance of specific traits explained by genetic factors. For example, a study with more than one million participants found that just 7\% of the variance was attributable to the genotyped variants [29]; a value which is notably lower than those obtained from twin studies.

There is also heterogeneity between age groups. In adolescents, heritability estimates range from 14 to $41 \%$ [30-33]. In young adults around $35 \%$ of the variance was explained by genetic factors [34]. In adults, heritability estimates range from 0.28 to 0.43 [27,35-37]. Regarding sex, different heritability estimates were reported for males and females in one study [24] but not in others [34,35,37].

The above cited literature consistently supports the substantial role of genetics in explaining individual differences for insomnia, although the magnitude of its impact varies between studies. There is even greater uncertainty about the specific variables that could potentially cause differences in the distribution of the genetic and environmental influences for insomnia. Women usually have a higher prevalence of insomnia than do men [38]. Gonadal steroid effects have been proposed as a potential explanation for these differences since sex differences in the prevalence of insomnia begins at puberty and increases during and after the stage of life at which females experience the menopause [39]. Nevertheless, mean differences in terms of symptoms, do not necessarily imply genetic and environment differences on the distribution of the variance. As stated above, just one study found sex-differences for the heritability estimates for insomnia [24]. This could be because many of the studies were relatively small and may have been underpowered to detect sex differences. Age is another variable that could affect the distribution of the variance on insomnia. Sleep changes throughout the life-span and insomnia is associated with aging $[3,40,41]$. Ethnicity is another variable that could potentially influence heritability estimates [42-44]. However, studies addressing these issues are not conclusive and most of them are limited to US samples. Related to the previous point, latitude, which is associated with hours of sun-light exposure, could also influence insomnia; for example, a study that compared sleep in Norway $\left(69^{\circ} \mathrm{N}\right)$ and Ghana $\left(5^{\circ} \mathrm{N}\right)$ found that lack of daylight was related to increased problems falling asleep and daytime fatigue. Seasonal differences in insomnia were found in Norway but not in Ghana [45].

Taking into account the wide variety of results across the studies this meta-analysis aimed to: 1) Estimate the mean heritability of insomnia; 2) Assess heterogeneity among twin studies on this phenotype; and 3) Search and analyse characteristics of the studies (moderator variables) that may explain the heterogeneity among such studies.

\section{Method}

This meta-analysis was pre-registered on the Open Science Framework (see for further information https://osf.io/5g839/). Furthermore, this systematic review and meta-analysis was conducted following the Preferred Reporting Items for Systematic Reviews and Meta-analyses (PRISMA) guidelines [46]. Supplementary Table 1 presents the PRISMA checklist for this systematic review.

\section{Eligibility criteria}

This meta-analysis focused on twin studies that provided an estimation of the heritability of insomnia up until March 2020. All studies that addressed insomnia or insomnia symptoms explicitly were included. Studies that included indirect measures that were not specifically referred to as insomnia symptoms (such as sleep disturbances, awakenings, or sleep onset difficulties) were not included. This was because they might have focused on variables other than insomnia such as general sleep quality or sleep apnoea. This approach also allowed consistency in the criterion for selection. Our search strategy included both objective and subjective measures of insomnia. Nonetheless, there were no studies using objective measures of insomnia that met the selection criteria.

As for the exclusion criteria, studies that did not use a twin sample to estimate heritability (e.g., GWAS) were excluded. Studies with a mean sample below 6 years old were also excluded since our focus was not on insomnia in young children, which may differ from that experienced at later stages of the life course as well as sleep characteristics and patterns (e.g., school timetables or daytime naps $[47,48])$. Only independent samples were included. Hence, when more than one publication reported the heritability of insomnia using the same sample (the same twin registry) we selected the publication with: 1) greater detail (e.g., estimates for men and women independently); 2) the larger sample; 3) estimates from univariate models (rather than multivariate models) or 4) a more recent publication date.

\section{Search strategy}

The scientific search was conducted from the 1 st to 30 th of March 2020 in PubMed (https://www.ncbi.nlm.nih.gov/pubmed/) and Web of Science (https://apps.webofknowledge.com/). The following keywords were used: insomnia AND monozygotic/dizygotic/twin*/heritability. No filters for dates or language were applied. However, conference papers were excluded since they often do not report all the required information and are typically published later as full research articles.

Applying these criteria, we found 82 and 144 results in PubMed and Web of Science respectively. Duplicates were removed yielding a total of 153 that were screened. Of those, 31 results were assessed and after applying the exclusion criteria, 10 articles were included in the meta-analysis (Fig. 1). As some studies reported heritability 

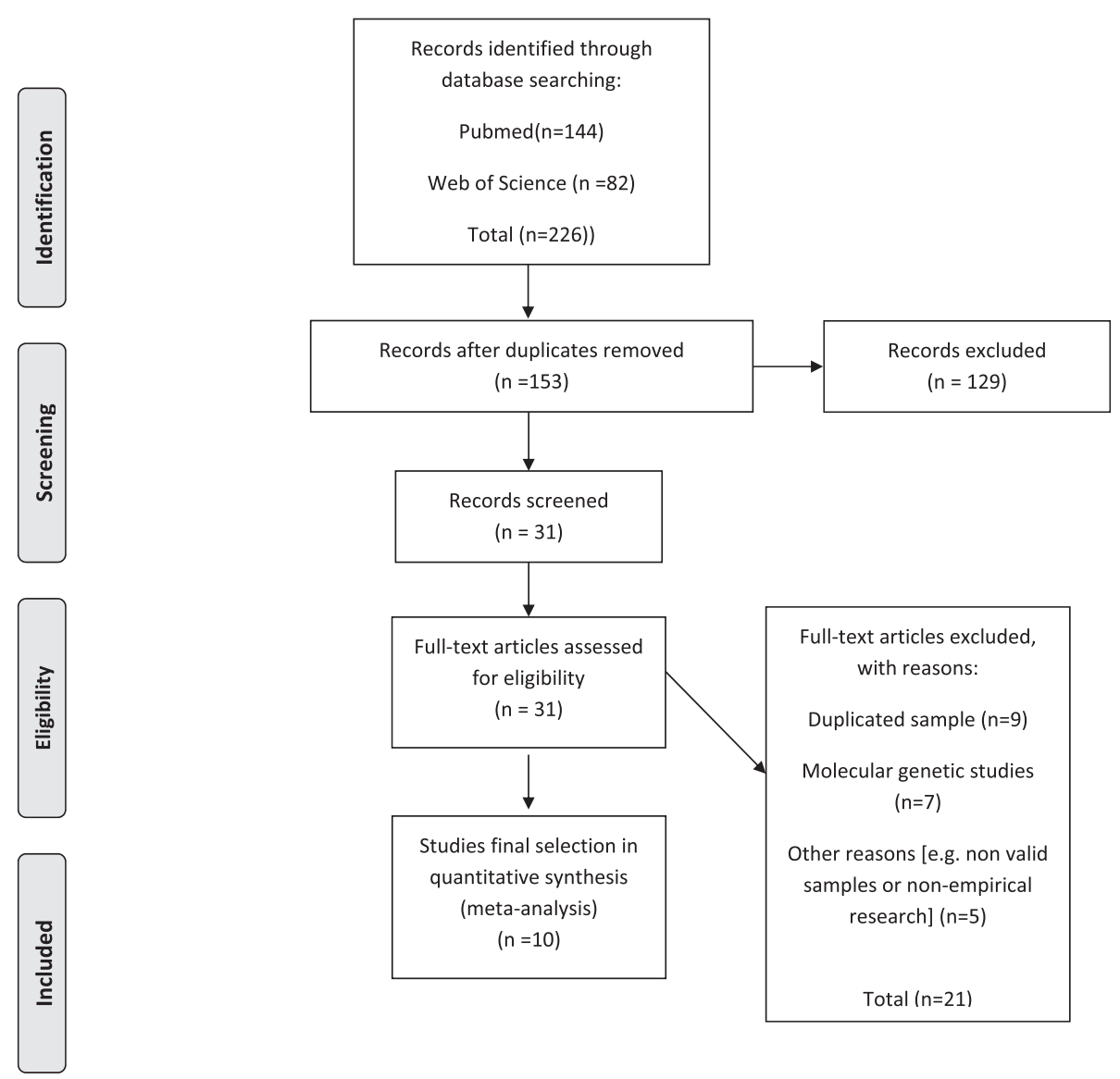

Fig. 1. Flow Chart of study selection process.

estimates for men and women independently, this yielded a total of 13 units of analyses.

\section{Data extraction}

For all the studies the following characteristics of the studies were extracted (as per a previous publication focusing on sleep quality and duration [49]).

- Mean age of the study sample with standard deviation

- Proportion of males and females

- Proportion of monozygotic (MZ), dizygotic (DZ), dizygotic opposite sex (DZOS) and non-twin siblings (if relevant)

- Country of origin of the study population

- Continent of origin of the study population

- Type of measure: single question or questions derived from a validated questionnaire/validated instrument

- Best fitting model (ACE, ADE, AE, CE or E)

- Number of twin/siblings pairs in the study

- Number of participants in the study

- MZ and DZ correlations

- Components of the variance for the full model: we entered the heritability $\left(\mathrm{h}^{2}\right)$ and shared environmental component $\left(c^{2}\right)$ under the ACE or ADE model. When the reported model was an ACE model, the estimate for A was entered in $h^{2}{ }_{-}$FULL and $C$ was entered in $C^{2}$ _FULL. When an ADE model was reported, we summed $A$ and $D$ and entered the sum in $h^{2}$ FULL and zero for $\mathrm{c}^{2}$ _FULL. If both univariate and multivariate analyses were presented, estimates from univariate models were included
- Components of the variance for the BEST model: When the best fitting model was an ACE model, we entered A for $\mathrm{h}^{2}{ }_{-}$BEST and $\mathrm{C}$ was entered for $C^{2}$ _FULL. When the best fitting model was an ADE, we entered the sum of $A$ and $D$ in $h^{2}{ }_{-}$BEST and zero in $\mathrm{c}^{2}$ _BEST. If the best fitting model was an $\mathrm{AE}$ (or CE or E) we entered zero for the component (or components) dropped and the significant components were entered as previously described

Data entry: All the studies were double coded by JJM-V and MRA, disagreements were solved by consensus by third reviewer (JRO or JSM). The results showed very satisfactory interrater reliability, with kappa coefficients ranging from 0.95 to $1(\mathrm{M}=0.97)$ for the categorical variables and intraclass correlations between 0.97 and $1(\mathrm{M}=0.98)$ for the continuous variables. Heath and colleagues [36] provided heritability estimates from five different questions (i.e., "initial insomnia; "disturbed sleep"; "anxious insomnia"; "depressed insomnia" and "sleep delay"). For this unit of analysis, the mean of the estimates for these five measures was calculated.

\section{Statistical analyses}

In this meta-analysis the outcome measures were monozygotic and dizygotic twin correlations (rMZ and rDZ, respectively), and estimates of heritability from the full and best fitting models $\left(h^{2}\right.$ FULL and $h^{2}$ BEST, respectively). These effect sizes were transformed into the Fisher's $\mathrm{Z}$ metric in order to normalize distributions and stabilize variances.

Separate meta-analyses were carried out for rMZ, rDZ, ${ }^{2}{ }_{-}$FULL and $\mathrm{h}^{2}$ BEST by assuming random-effects models, as heterogeneity 
among the effect sizes was expected. The inverse variance method was used to weigh each effect size. The variance was equal to the sum of the sampling variance and the between-studies variance, as estimated by restricted maximum likelihood [50]. For each metaanalysis, a mean effect size was obtained and a 95\%CI was constructed with the improved method developed by Hartung and Knapp [51,52]. To facilitate the interpretation of the results, the mean effect sizes and their confidence limits (calculated on Fisher's Z transformed effect sizes) were back transformed into a Pearson correlation metric. To check for variability among effect sizes, the Cochran's Q-statistic and the $I^{2}$ index (values of 0\%, 25\%, 50\%, and 75\% representing no, low, moderate, and high heterogeneity, respectively) were used [53]. For each meta-analysis, a forest plot was also constructed.

Publication bias was assessed by constructing funnel plots with the trim-and-fill method which consists of imputing missing effect sizes to achieve symmetry [54]. The Egger's regression test was also applied [55]. A statistically significant result for the Egger test $(\mathrm{p}<.10)$ was evidence of publication bias. Using $\mathrm{p}<.10$ in place of the usual $\mathrm{p}<.05$ is due to the Egger test has low statistical power with a small number of studies $(\mathrm{k}<20)$, as it is the case [56].

Finally, in order to explain the heterogeneity among the effect sizes, meta-regressions and weighted ANOVAs for continuous and categorical moderators respectively, were applied by assuming a mixed-effects model for each meta-analysis with at least 10 effect sizes. An improved $F$ statistic developed by Knapp and Hartung $[57,58]$ was applied for testing the statistical significance of each moderator. $Q_{\mathrm{E}}$ and $Q_{\mathrm{W}}$ statistics were computed to test the model misspecification for meta-regressions and weighted ANOVAs, respectively, and an estimate of the proportion of variance explained by each moderator, $R^{2}$, was also calculated [59]. All statistical analyses were conducted with metafor program in $\mathrm{R}$ [60].

\section{Results}

\section{Characteristics of the included studies}

All the studies used the classical twin design, which make use of the difference between MZ and DZ twins within-pair correlations, to estimate the genetic and environmental influences on insomnia. One study reported data from women only [27] and another only men [26]. Studies were carried out using samples from: North America (5), Europe (4) and Oceania (1). Actually, just four countries provided data: USA (5), UK (3), Finland (1) and Australia (1). Three studies had data available for males and females separately and therefore, they provided two units of analyses (i.e., one for males and one for females) [24,35,37]. Gregory et al. [34], Lind et al.
[24], and Barclay et al. [30], reported heritability estimates from different waves of data collection. The wave with larger number of participants was selected (Table 1).

\section{Mean effect size and heterogeneity}

Table 2 presents the results of the meta-analyses carried out for twin correlations (rMZ and rDZ) and variance components $\left(h^{2}\right.$ FULL and $h^{2}$ _BEST). Fig. 2 displays the forest plots for rMZ, rDZ, $\mathrm{h}^{2}{ }_{-}$FULL and $\mathrm{h}^{2}{ }_{\text {BEST. }}$.

Thirteen studies reported the MZ and DZ correlations and the heritability estimates from the best fitting model. There were seven heritability estimates from full models. The mean effect size for $\mathrm{rMZ}$ was $\mathrm{ES}_{+}=0.37(95 \% \mathrm{CI}=0.31,0.43)$, and for $\mathrm{rDZ}$ was $\mathrm{ES}_{+}=0.15$ $(95 \% \mathrm{CI}=0.12,0.18)$. Similar mean effect sizes were found for heritability estimates, being $\mathrm{ES}_{+}=0.41(95 \% \mathrm{CI}=0.32,0.49)$ for $\mathrm{h}^{2}{ }_{\text {_FULL }}$ and $\mathrm{ES}_{+}=0.39(95 \% \mathrm{CI}=0.32,0.44)$ for $\mathrm{h}^{2}{ }_{-}$BEST.

Great heterogeneity among the effect sizes for rMZ, rDZ, $\mathrm{h}^{2}$ _FULL and $\mathrm{h}^{2}$ _BEST was found $\left(\mathrm{I}^{2}>75 \%\right.$ and $p<.0001$, except for rDZ where $I^{2}=48 \%$ ) (see Table 2 ). This considerable heterogeneity is also reflected in the forest plots (see Fig. 2).

\section{Analysis of publication bias}

Publication bias was analysed by constructing a funnel plot and assessing its asymmetry with the trim-and-fill method and the Egger test.

Fig. 3 presents the funnel plots for rMZ, rDZ, $\mathrm{h}^{2}$ FULL and $\mathrm{h}^{2}{ }_{-}$BEST. Applying the trim-and-fill method, no effect sizes had to be imputed for MZ and DZ twin correlations and $h^{2} \_$BEST (see Fig. 3A, B and 3D). For the $\mathrm{h}^{2}$ FULL, an additional effect size

Table 2

Mean effect sizes, 95\% confidence intervals, and heterogeneity statistics of twin correlations and variance components.

\begin{tabular}{lllllll}
\hline Statistic & $k$ & $\mathrm{ES}_{+}$ & $\frac{95 \% \mathrm{CI}}{\mathrm{LL} \mathrm{UL}}$ & $Q$ & $p$ & $I^{2}$ \\
\hline$r_{M Z}$ & 13 & 0.37 & 0.310 .43 & 90.18 & $<0.0001$ & 89.23 \\
$r_{D Z}$ & 13 & 0.15 & 0.120 .18 & 25.23 & 0.014 & 48.05 \\
$h^{2}$ _FULL & 7 & 0.41 & 0.320 .49 & 181.78 & $<0.0001$ & 97.80 \\
$h^{2} \_$BEST & 13 & 0.39 & 0.320 .44 & 549.39 & $<0.0001$ & 98.77 \\
\hline
\end{tabular}

$r=$ correlation. $\mathrm{MZ}=$ monozygotic twins. $\mathrm{DZ}=$ dizygotic twins. $h^{2}$ _FULL $=$ heritability estimates from the full model. $h^{2} \_$BEST $=$heritability estimates from the best fitting model. $k=$ number of studies. $\mathrm{ES}_{+}=$mean effect size estimate. LL and UL: lower and upper limits of the 95\% confidence interval for $\mathrm{ES}_{+}$. $Q=$ Cochran's heterogeneity $Q$ statistic; $Q$ statistic has $k-1$ degrees of freedom. $p=$ probability level for the $Q$ statistic. $I^{2}=$ heterogeneity index.

Table 1

Main characteristics of the studies included in the meta-analysis.

\begin{tabular}{|c|c|c|c|c|c|c|c|}
\hline & Study & $\mathrm{N}$ & Validated questionnaire & Continent (Country) & Age & \%Males & $\mathrm{H}^{2}$ Insomnia \\
\hline 1 & Heath et al., 1990 [36] & 3810 pairs & NO & Oceania (Australia) & 1 & 36 & 0.34 \\
\hline 2 & McCarren et al., 1994 [26] & 2825 pairs & YES & America (USA) & l & 100 & 0.28 \\
\hline 3 & Watson et al., 2006 [25] & 1870 pairs & NO & America (USA) & $\overline{\mathrm{x}}=32$ & 39 & 0.57 \\
\hline \multirow[t]{2}{*}{4} & Hublin et al., 2011 [35] & 4545 pairs & NO & Europe (Finland) & $\mathrm{A}: \overline{\mathrm{x}}=43.9$ & A: 100 & A:0.42 \\
\hline & & & & & $\mathrm{B}: \overline{\mathrm{x}}=43.9$ & $\mathrm{~B}: 0$ & B:0.42 \\
\hline \multirow[t]{2}{*}{5} & Drake et al., 2011 [37] & 1782 participants & NO & America (USA) & $\mathrm{A}: \overline{\mathrm{x}}=22.5$ & A: 100 & A:0.43 \\
\hline & & & & & $\mathrm{B}: \overline{\mathrm{x}}=22.5$ & $\mathrm{~B}: 0$ & B:0.55 \\
\hline 6 & Hur et al., 2012 [27] & 3758 participants & NO & Europe (UK) & $\overline{\mathrm{x}}=50$ & 0 & 0.28 \\
\hline 7 & Barclay et al., 2015 [30] & 2822 participants & NO & America (USA) & $\overline{\mathrm{x}}=12$ & 46 & 0.33 \\
\hline \multirow[t]{2}{*}{8} & Lind et al., 2015 [24] & 7500 participants & NO & America (USA) & $A: \bar{x}=29.3$ & A:100 & A:0.28 \\
\hline & & & & & $\mathrm{B}: \overline{\mathrm{x}}=29.3$ & $\mathrm{~B}: 0$ & B:0.30 \\
\hline 9 & Gregory et al., 2016 [34] & 1556 participants & YES & Europe (UK) & $\overline{\mathrm{x}}=20.3$ & 38 & 0.35 \\
\hline 10 & Madrid-Valero et al., 2020 [33] & 10,222 participants & YES & Europe (UK) & $\overline{\mathrm{x}}=16.3$ & 44.8 & 0.41 \\
\hline
\end{tabular}

A: males; B: females.

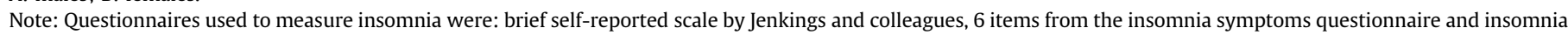
severity index for McCarren et al., 1994, Gregory et al., 2016 and Madrid-Valero et al., 2020 respectively. 
A

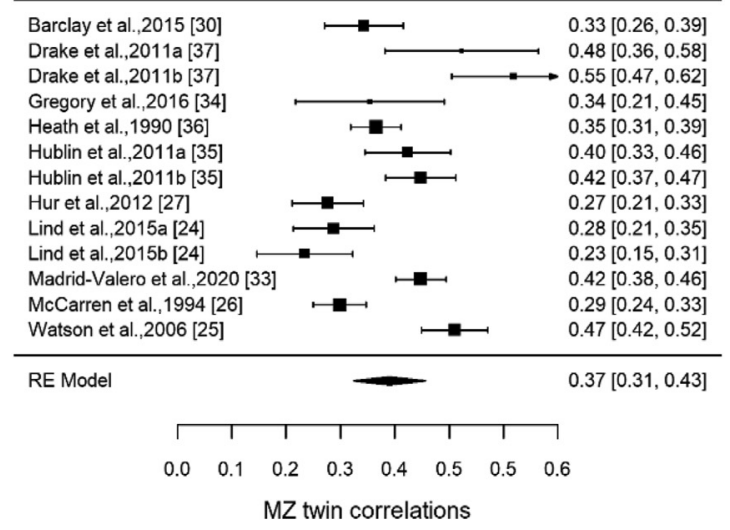

C

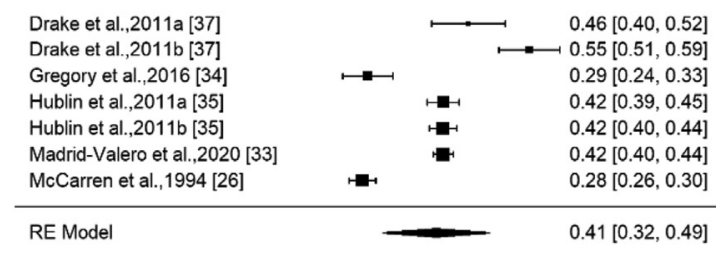

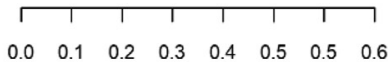

$\mathrm{H}^{2} \mathrm{FULL}$

\section{B}

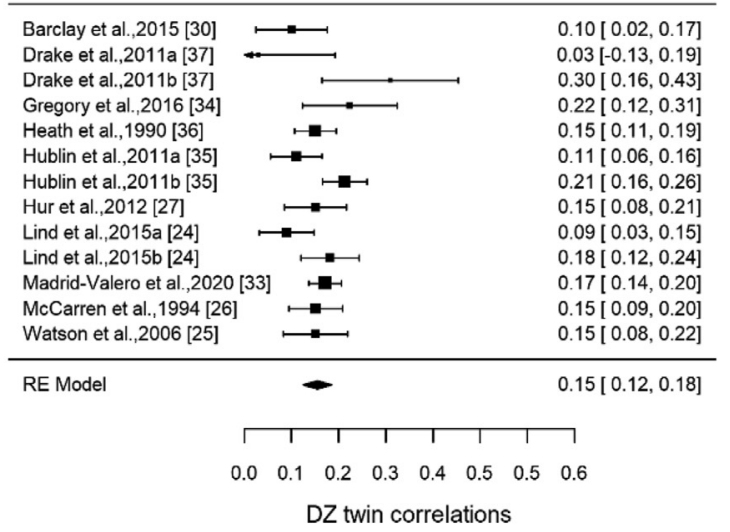

D

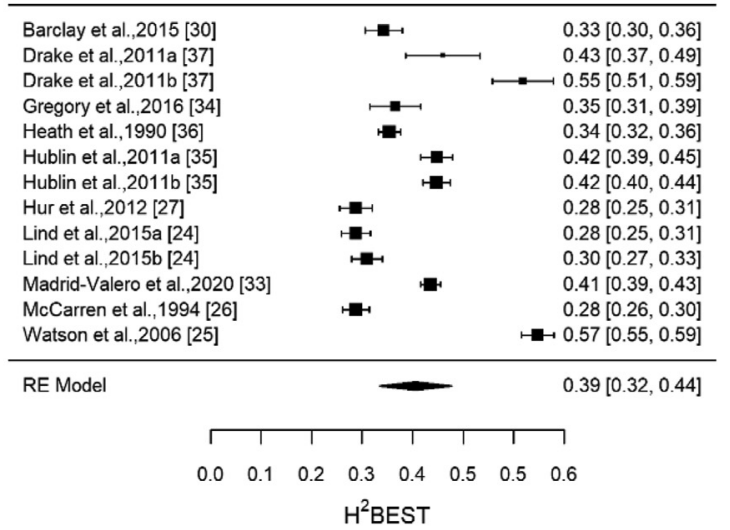

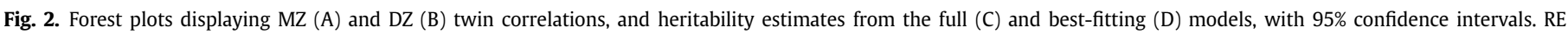
(Random-Effects) Model refers to the statistical model assumed in the calculations.

estimate was imputed on the left side to the original set of estimates to achieve symmetry in the funnel plot (see Fig. 3C). The adjusted mean effect size, once corrected by publication bias, was $\mathrm{ES}_{\mathrm{adj}}=0.40(95 \% \mathrm{CI}=0.31,0.49)$. Compared with the original mean effect size obtained with the seven studies $\left(\mathrm{ES}_{+}=0.41\right)$, the adjusted mean effect size barely changed, with a negligible decrease of $2.40 \%$.

Non-significant results for the interception were obtained from the Egger test for $\mathrm{MZ}$ twin correlations $(t(11)=1.00 ; p=.338)$, DZ twin correlations $(t(11)=0.13 ; p=.898)$, heritability estimates from the full model $(t(5)=1.05 ; p=.343)$ and heritability estimates from the best fitting model $(t(11)=1.30 ; p=.219)$.

\section{Analyses of moderator variables}

The large variability found among the effect sizes led to the analysis of potential moderator variables. These analyses were applied to those meta-analyses with at least 10 effects sizes, i.e., rMZ, rDZ, and $\mathrm{h}^{2} \_$BEST.

Table 3 presents the results of the simple meta-regressions performed on several continuous moderators. We were not able to analyse quantitative moderators for $\mathrm{rMZ}$ and $\mathrm{rDZ}$ due to the lack of available information. None of the analysed moderators reached a statistically significant association with the $\mathrm{h}^{2}$ _BEST.
Continent where the study took place and the use of a validated questionnaire were also analysed, as categorical moderators, by means of the weighted ANOVAs models. Table 4 presents those results. None of them reached a statistically significant association with $\mathrm{MZ}$ and $\mathrm{DZ}$ twin correlations or heritability estimates from the best fitting model.

\section{Discussion}

This study meta-analysed twin studies that estimated the magnitude of genetic and environmental influences on insomnia. As expected, we found that genetic factors play a substantial role in explaining variability for insomnia. The mean effect size for genetic influences on insomnia was 0.39 (95\% CI: 0.32,.44) from the best fitting models. This result is similar to the heritability estimates for sleep quality and sleep duration reported in another recent metaanalysis by our group [49]. Furthermore, our results also reveal high heterogeneity among studies.

Regarding the distribution of the variance, non-shared environmental influences were the main source of variation with values ranging from 0.43 to 0.72 . Genetic influences were also substantial with values ranging from 0.28 to 0.57 . In contrast, shared-environmental influences had a negligible impact in all the studies. This is in line with the wider behavior genetic 

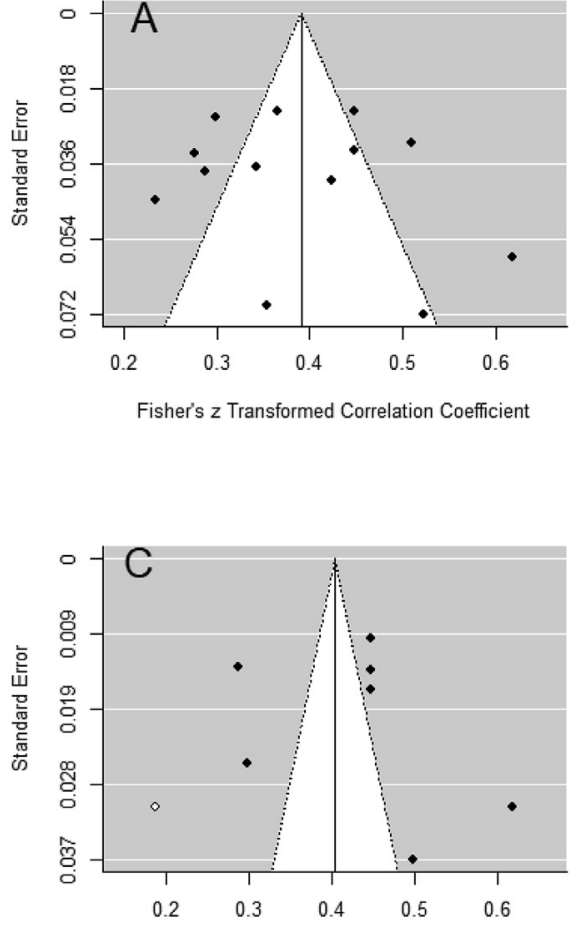

Fisher's z Transformed Correlation Coefficient

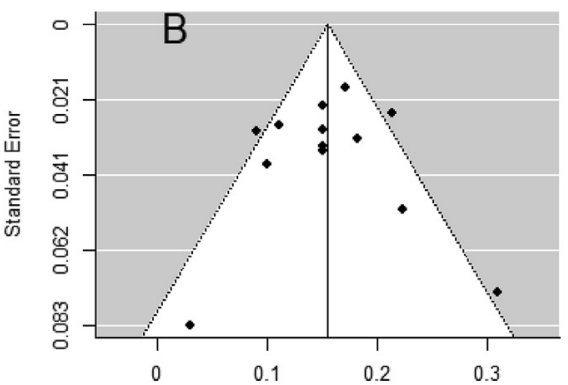

Fisher's z Transformed Correlation Coefficient

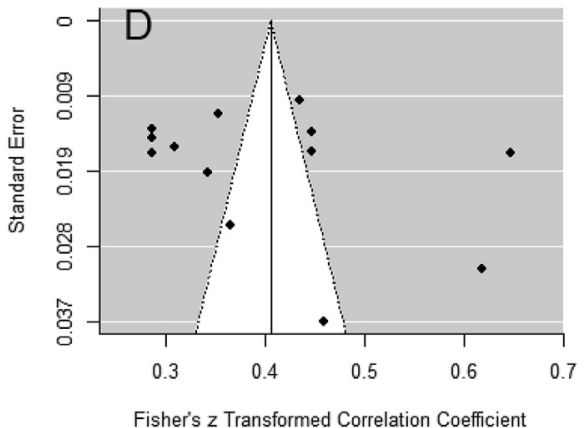

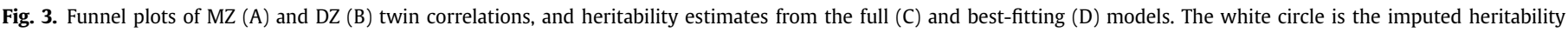
estimate by means of the Duval and Tweedie's trim and-fill method.

\section{Table 3}

Results of the simple meta-regressions applied on heritability estimates from the best fitting model, taking continuous moderator variables as predictors.

\begin{tabular}{lllllll}
\hline Predictor variable & $k$ & $b_{\mathrm{j}}$ & $F$ & $p$ & $Q_{\mathrm{E}}$ & $R^{2}$ \\
\hline$h^{2} \_$BEST & & & & & & \\
Mean age (years) & 11 & -0.001 & 0.07 & 0.805 & $460.29 * * *$ & 0 \\
Gender (\% male) & 13 & -0.001 & 0.35 & 0.568 & $528.69^{* * *}$ & 0 \\
\% of MZ & 13 & 0.004 & 1.04 & 0.329 & $541.03^{* * *}$ & 0 \\
\% of DZ & 13 & -0.004 & 1.09 & 0.320 & $539.84^{* * *}$ & 0 \\
\% of DOS & 9 & -0.004 & 0.95 & 0.363 & $345.57^{* * *}$ & 0 \\
\hline
\end{tabular}

$\mathrm{MZ}=$ monozygotic twins. $\mathrm{DZ}=$ dizygotic twins. $\mathrm{DOS}=$ dizygotic twins, opposite sex. $k=$ number of studies. $b_{\mathrm{j}}=$ regression coefficient of each predictor. $F=$ KnappHartung's statistic for testing the significance of the predictor (the degrees of freedom for this statistic are one for the numerator and $k-2$ for the denominator). $p=$ probability level for the $F$ statistic. $Q_{\mathrm{E}}=$ statistic for testing the model misspecification. $R^{2}=$ proportion of variance accounted for by the predictor. $* * * p<.0001 . * p<.01$.

literature, where $C$ usually decreases with age and becomes undetectable [61] for many traits. This is also consistent with the aforementioned meta-analysis on sleep quality and sleep duration, where just two studies on sleep duration reported commonshared environmental influences and both influences were of small magnitude [49].

GWAS have also confirmed the role of genes in explaining interindividual differences on this trait. For example, Jansen et al. [29], in a study using more than one million participants, estimated SNP-based heritability for insomnia at $7 \%$. This value is notably lower than those obtained from twin studies reported here. Such a divergence in estimates between twin and SNP-based studies is found frequently in different traits and represents the issue of so-
Table 4

Results of the weighted ANOVAs applied on monozygotic twin correlations, dizygotic twin correlations and heritability estimates from the best fitting model, taking qualitative moderator variables as independent variables.

\begin{tabular}{|c|c|c|c|c|c|}
\hline \multirow[t]{2}{*}{ Predictor variable } & \multirow[t]{2}{*}{$k$} & \multirow[t]{2}{*}{$\mathrm{ES}_{+}$} & \multicolumn{2}{|c|}{$95 \% \mathrm{CI}$} & \multirow[t]{2}{*}{ ANOVA results } \\
\hline & & & LL & $\mathrm{LU}$ & \\
\hline \multicolumn{6}{|l|}{$r_{M Z}$} \\
\hline \multicolumn{6}{|r|}{$F_{210}=0.03, p=.972$} \\
\hline N. America & 7 & 0.38 & 0.29 & 0.46 & $R^{2}=0$ \\
\hline Europe & 5 & 0.37 & 0.27 & 0.47 & $Q_{\mathrm{W}}(10)=86.11, p<.001$ \\
\hline Oceania & 1 & 0.35 & 0.11 & 0.55 & \\
\hline Validated questionnaire: & & & & & $F_{1,11}=0.15, p=.702$ \\
\hline No & 10 & 0.38 & 0.31 & 0.44 & $R^{2}=0$ \\
\hline Yes & 3 & 0.35 & 0.22 & 0.47 & $Q_{\mathrm{W}}(11)=89.99, p<.001$ \\
\hline \multicolumn{6}{|l|}{$r_{D Z}$} \\
\hline \multicolumn{6}{|l|}{ Continent: } \\
\hline N. America & 7 & 0.14 & 0.09 & 0.19 & $R^{2}=0$ \\
\hline Europe & 5 & 0.17 & 0.12 & 0.22 & $Q_{\mathrm{W}}(10)=22.12, p=.015$ \\
\hline Oceania & 1 & 0.15 & 0.05 & 0.25 & \\
\hline Validated questionnaire: & & & & & $F_{1,11}=0.61, p=.452$ \\
\hline No & 10 & 0.15 & 0.11 & 0.19 & $R^{2}=0$ \\
\hline Yes & 3 & 0.17 & 0.11 & 0.23 & $Q_{\mathrm{w}}(11)=23.87, p=.013$ \\
\hline \multicolumn{6}{|c|}{ 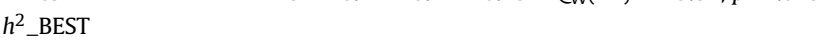 } \\
\hline Continent: & & & & & $F_{2,10}=0.14, p=.873$ \\
\hline N. America & 7 & 0.39 & 0.30 & 0.49 & $R^{2}=0$ \\
\hline Europe & 5 & 0.38 & 0.26 & 0.48 & $Q_{W}(10)=523.28, p<.001$ \\
\hline Oceania & 1 & 0.34 & 0.07 & 0.56 & \\
\hline Validated questionnaire: & & & & & $F_{1,11}=0.49, p=.497$ \\
\hline No & 10 & 0.39 & 0.32 & 0.47 & $R^{2}=0$ \\
\hline Yes & 3 & 0.35 & 0.21 & 0.48 & $Q_{W}(11)=547.30, p<.001$ \\
\hline
\end{tabular}

$k=$ number of studies. ES $=$ average effect size estimate. $\mathrm{LL}$ and LU $=$ lower and upper 95\% confidence limits for $\mathrm{ES}_{+} . F=$ Knapp-Hartung's statistic for testing the significance of the moderator variable. $Q_{W}=$ statistic for testing the model misspecification. $R^{2}=$ proportion of variance accounted for by the moderator. 
called 'missing heritability'. Proposed explanations for this difference in heritability estimated from twin studies and those assessing genetic variants (i.e., SNPs) include that SNP methods fail to capture the influence of many common variants of very small effect and also of rare variants with large effects. Furthermore, twin studies may involve specific effects such as gene-environment correlation that are not captured by SNPbased methods [62-64]. In the aforementioned study more than 200 loci implicating 956 genes were identified. This study also supported the link between sleep disorders and psychiatric disorders, findings that have extensively reported using twin samples [33,65-67]. Other GWAS studies have also found significant genetic correlations between insomnia and alcohol use, nicotine use and opioid use [68]. A Recent GWAS also demonstrated that genetic variation associated with brain structure also affects insomnia [69]. Research about the aetiology of insomnia is essential for many reasons. The more we know the more we advance in our endeavor of developing preventing strategies as well as more accurate and effective treatments, such as tailored interventions. It has been proposed that there might be two types of insomnia, one with a more biological origin [70]. If this theory is supported it could suggest that different treatments should be used for each type of insomnia and genetic research will be essential in clarifying this. Finally, these findings are also relevant from a clinical perspective. It has been demonstrated that perceptions about the aetiology of a disorder could influence perceptions and attitudes regarding different treatments and clinicians' empathy [71,72].

As stated above, there was significant heterogeneity among studies. Nevertheless, none of the moderators considered (e.g., age, sex, continent, or type of measure) could explain the variability among the studies. Age and sex are two of the most studied variables associated with insomnia. Female sex is a risk factor for insomnia and poor sleep quality, which also appears to worsen with increasing age $[38,40]$. Despite the scientific literature consistently supporting these findings, our results did not provide any evidence for a significant effect of age or sex. This failure to detect the effect of these moderators could be due to the limited number of studies included in the meta-analysis or because most of the studies did not specifically assess age and sex in their analyses. Results from the studies which reported heritability estimates for males and females separately revealed that estimates are quite similar. These results are consistent with other sleep phenotypes, specifically sleep duration and sleep quality where no moderators were found in a previous metaanalysis [49].

There was no evidence of publication bias. Publication bias is unlikely for twin studies since they possess characteristics such as large samples and relevant effect sizes (typically heritability estimates are around 30-50\%). Moreover, low heritability estimates are as interesting as high heritability values [61].

\section{Strengths and limitations}

This study has several strengths such as the use of a metaanalytic approach to estimate the mean effect size of heritability estimates. Furthermore, as previously mentioned, twin studies typically use large twin samples, and high and low heritability estimates are equally interesting. All the studies included here flagged significant heritability estimates for insomnia. In addition to this, both best fitting and full models were meta-analysed, since estimates from best fitting models could be affected by the sample size. We found very similar results using both approaches. Despite these strengths this study also has some limitations. First, the number of studies was limited: only 10 independent samples (13 units of analyses) were included. These studies came from just four different countries which means that their representativeness could be limited. Furthermore, some studies did not report all of the statistics. For example, while all units of analyses were available for the best fitting model, just 7 units of analyses reported data for the full model which hampered the comparison between full and best fitting models. Additionally, most of the studies did not provide data for males and females separately and the same applies for different age ranges, which hampered the search for moderators. In light of these factors, we encourage behavior genetic researchers to report data from all models and, if relevant, sex comparisons and sex-limitation models. Regarding the measurement of insomnia, it is important to highlight that no studies estimated the heritability of insomnia using objective methods (this is noteworthy, although unsurprising given that insomnia is largely considered a subjective complaint - best assessed using subjective measures). Finally, despite the large heterogeneity demonstrated by the effect sizes, we have not been able to identify moderators of such variability. This could be due to the limited number of studies included in our meta-analysis. Other hidden moderators not reported in the studies could also affect heritability estimates of insomnia. Therefore, the small number of studies invites a cautious interpretation of the results of moderator analyses.

\section{Conclusions}

There is significant heterogeneity in genetic and environmental influences on insomnia from twin samples. Around $40 \%$ of the variance in insomnia is explained by genetic factors. Further research using different samples is needed (especially in those populations with unique characteristics for sleep such as a specific geography or climate) to identify possible moderators.

\section{Conflicts of interest}

The authors declare no conflict of interest. Alice Gregory is an advisor for a project sponsored by Johnson's Baby. She receives royalties for two books Nodding Off (Bloomsbury Sigma, 2018) and The Sleepy Pebble (Flying Eye). She is a regular contributor to BBC Focus magazine and has contributed to other outlets (such as The Conversation, The Guardian and Balance Magazine). She occasionally receives sample products related to sleep (e.g., blue light blocking glasses) and has given a paid talk to a banking and wealth management company.

\section{Practice points}

1 This review confirms that insomnia is moderately influenced by genetic factors

2 Results from this study highlight that non-shared environmental influences are the main source of variance for insomnia

3 Common shared environmental factors have a negligible impact on insomnia in participants who are older than six years of age 


\section{Research agenda}

1 Twin studies should always provide information from all models and test for sex differences where there is power to do so

2 Further research is needed focusing on participants from other locations since twin studies on insomnia come from just four countries

3 More studies are needed to elucidate the role of moderators such as age and sex on the distribution of the variance in insomnia

\section{Appendix A. Supplementary data}

Supplementary data to this article can be found online at https://doi.org/10.1016/j.smrv.2021.101437.

\section{References}

[1] Morin CM, Drake CL, Harvey AG, Krystal AD, Manber R, Riemann D, et al. Insomnia disorder. Nat Rev Dis Primers 2015;1:15026.

[2] Ohayon MM, Sagales T. Prevalence of insomnia and sleep characteristics in the general population of Spain. Sleep Med 2010;11(10):1010-8.

[3] Ohayon MM. Epidemiology of insomnia: what we know and what we still need to learn. Sleep Med Rev 2002;6(2):97-111.

[4] Morin CM, LeBlanc M, Bélanger L, Ivers H, Mérette C, Savard J. Prevalence of insomnia and its treatment in Canada. Can J Psychiatr 2011;56(9):540-8.

[5] Cao XL, Wang SB, Zhong BL, Zhang L, Ungvari GS, Ng CH, et al. The prevalence of insomnia in the general population in China: a meta-analysis. PloS One 2017;12(2):e0170772.

[6] Roth T. Insomnia: definition, prevalence, etiology, and consequences. J Clin Sleep Med 2007;3(5 Suppl):S7-10.

[7] Fortier-Brochu E, Beaulieu-Bonneau S, Ivers H, Morin CM. Insomnia and daytime cognitive performance: a meta-analysis. Sleep Med Rev 2012;16(1):83-94.

[8] Fortier-Brochu E, Morin CM. Cognitive impairment in individuals with insomnia: clinical significance and correlates. Sleep 2014;37(11):1787-98.

[9] He Q Zhang P, Li G, Dai H, Shi J. The association between insomnia symptoms and risk of cardio-cerebral vascular events: a meta-analysis of prospective cohort studies. Eur J Prev Cardiol 2017;24(10):1071-82.

[10] Zheng B, Yu C, Lv J, Guo Y, Bian Z, Zhou M, et al. Insomnia symptoms and risk of cardiovascular diseases among 0.5 million adults: a 10-year cohort. Neurology 2019;93(23):e2110-20.

[11] Tang NK. Insomnia Co-occurring with chronic pain: clinical features, interaction, assessments and possible interventions. Rev Pain 2008;2(1):2-7.

[12] Mullington JM, Simpson NS, Meier-Ewert HK, Haack M. Sleep loss and inflammation. Best Pract Res Clin Endocrinol Metabol 2010;24(5):775-84.

[13] Garbarino S, Magnavita N, Guglielmi O, Maestri M, Dini G, Bersi FM, et al. Insomnia is associated with road accidents. Further evidence from a study on truck drivers. PloS One 2017;12(10):e0187256.

[14] Hertenstein E, Feige B, Gmeiner T, Kienzler C, Spiegelhalder K, Johann A, et al. Insomnia as a predictor of mental disorders: a systematic review and metaanalysis. Sleep Med Rev 2019;43:96-105.

[15] Li L, Wu C, Gan Y, Qu X, Lu Z. Insomnia and the risk of depression: a metaanalysis of prospective cohort studies. BMC Psychiatr 2016;16(1):375.

[16] Alvaro PK, Roberts RM, Harris JK. A systematic review assessing bidirectionality between sleep disturbances, anxiety, and depression. Sleep 2013;36(7):1059-68.

[17] Daley M, Morin CM, LeBlanc M, Grégoire JP, Savard J. The economic burden of insomnia: direct and indirect costs for individuals with insomnia syndrome, insomnia symptoms, and good sleepers. Sleep 2009;32(1):55-64.

[18] Wickwire EM, Tom SE, Scharf SM, Vadlamani A, Bulatao IG, Albrecht JS. Untreated insomnia increases all-cause health care utilization and costs among Medicare beneficiaries. Sleep 2019;42(4).

[19] Trauer JM, Qian MY, Doyle JS, Rajaratnam SM, Cunnington D. Cognitive behavioral therapy for chronic insomnia: a systematic review and metaanalysis. Ann Intern Med 2015;163(3):191-204.

[20] Watanabe N, Furukawa TA, Shimodera S, Katsuki F, Fujita H, Sasaki M, et al. Cost-effectiveness of cognitive behavioral therapy for insomnia comorbid with depression: analysis of a randomized controlled trial. Psychiatr Clin Neurosci 2015;69(6):335-43.

[21] Health NIo. National institutes of health state of the science conference statement on manifestations and management of chronic insomnia in adults, june 13-15, 2005. Sleep 2005;28(9):1049-57.

[22] Odintsova VV, Willemsen G, Dolan CV, Hottenga JJ, Martin NG, Slagboom PE, et al. Establishing a twin register: an invaluable resource for (behavior) genetic, epidemiological, biomarker, and 'omics' studies. Twin Res Hum Genet 2018;21(3):239-52.

[23] Knopik VS, Neiderhiser JM, DeFries JC, Plomin R. Behavioral genetics. 7th ed. New York: Worth Publishers; 2017.

*[24] Lind MJ, Aggen SH, Kirkpatrick RM, Kendler KS, Amstadter AB. A longitudinal twin study of insomnia symptoms in adults. Sleep 2015;38(9):1423-30.

*[25] Watson NF, Goldberg J, Arguelles L, Buchwald D. Genetic and environmental influences on insomnia, daytime sleepiness, and obesity in twins. Sleep 2006;29(5):645-9.

*[26] McCarren M, Goldberg J, Ramakrishnan V, Fabsitz R. Insomnia in Vietnam era veteran twins: influence of genes and combat experience. Sleep 1994;17(5): 456-61.

*[27] Hur Y-M, Burri A, Spector TD. The genetic and environmental structure of the covariation among the symptoms of insomnia, fatigue, and depression in adult females. Twin Res Hum Genet 2012;15(6):720-6.

[28] Visscher PM, Hill WG, Wray NR. Heritability in the genomics era-concepts and misconceptions. Nat Rev Genet 2008;9(4):255-66.

[29] Jansen PR, Watanabe K, Stringer S, Skene N, Bryois J, Hammerschlag AR, et al. Genome-wide analysis of insomnia in 1,331,010 individuals identifies new risk loci and functional pathways. Nat Genet 2019;51(3):394-403.

*[30] Barclay NL, Gehrman PR, Gregory AM, Eaves LJ, Silberg JL. The heritability of insomnia progression during childhood/adolescence: results from a longitudinal twin study. Sleep 2015;38(1):109-18.

[31] Gehrman PR, Meltzer LJ, Moore M, Pack AI, Perlis ML, Eaves LJ, et al. Heritability of insomnia symptoms in youth and their relationship to depression and anxiety. Sleep 2011;34(12):1641-6.

[32] Taylor MJ, Gregory AM, Freeman D, Ronald A. Do sleep disturbances and psychotic-like experiences in adolescence share genetic and environmental influences? J Abnorm Psychol 2015;124(3):674-84.

*[33] Madrid-Valero JJ, Ronald A, Shakeshaft N, Schofield K, Malanchini M, Gregory AM. Sleep quality, insomnia, and internalizing difficulties in adolescents: insights from a twin study. Sleep 2020;43(2).

*[34] Gregory AM, Rijsdijk FV, Eley TC, Buysse DJ, Schneider MN, Parsons M, et al. A longitudinal twin and sibling study of associations between insomnia and depression symptoms in young adults. Sleep 2016;39(11):1985-92.

*[35] Hublin C, Partinen M, Koskenvuo M, Kaprio J. Heritability and mortality risk of insomnia-related symptoms: a genetic epidemiologic study in a population-based twin cohort. Sleep 2011;34(7):957-64.

*[36] Heath AC, Kendler KS, Eaves LJ, Martin NG. Evidence for genetic influences on sleep disturbance and sleep pattern in twins. Sleep $1990 ; 13(4): 318-35$.

*[37] Drake CL, Friedman NP, Wright Jr KP, Roth T. Sleep reactivity and insomnia: genetic and environmental influences. Sleep 2011;34(9):1179-88.

[38] Zhang B, Wing YK. Sex differences in insomnia: a meta-analysis. Sleep 2006;29(1):85-93.

[39] Johnson EO, Roth T, Schultz L, Breslau N. Epidemiology of DSM-IV insomnia in adolescence: lifetime prevalence, chronicity, and an emergent gender difference. Pediatrics 2006;117(2):e247-56.

[40] Ohayon MM, Carskadon MA, Guilleminault C, Vitiello MV. Meta-analysis of quantitative sleep parameters from childhood to old age in healthy individuals: developing normative sleep values across the human lifespan. Sleep 2004;27(7):1255-73.

[41] Miner B, Kryger MH. Sleep in the aging population. Sleep Med Clin 2017;12(1):31-8.

[42] Grandner MA, Petrov ME, Rattanaumpawan P, Jackson N, Platt A, Patel NP. Sleep symptoms, race/ethnicity, and socioeconomic position. J Clin Sleep Med 2013;9(9):897-905 [A-D].

[43] Roberts RE, Roberts CR, Chan W. Ethnic differences in symptoms of insomnia among adolescents. Sleep 2006;29(3):359-65.

[44] Ruiter ME, DeCoster J, Jacobs L, Lichstein KL. Sleep disorders in african Americans and caucasian Americans: a meta-analysis. Behav Sleep Med 2010;8(4):246-59.

[45] Friborg O, Bjorvatn B, Amponsah B, Pallesen S. Associations between seasonal variations in day length (photoperiod), sleep timing, sleep quality and mood: a comparison between Ghana ( $5^{\circ}$ ) and Norway (69 ${ }^{\circ}$ ). J Sleep Res 2012;21(2): $176-84$.

[46] Moher D, Liberati A, Tetzlaff J, Altman D, Grp P. Preferred reporting items for systematic reviews and meta-analyses: the PRISMA statement. J Clin Epidemiol 2009;62(10):1006-12.

[47] Hirshkowitz M, Whiton K, Albert SM, Alessi C, Bruni O, DonCarlos L, et al. National Sleep Foundation's sleep time duration recommendations: methodology and results summary. Sleep Health 2015;1(1):40-3.

[48] Hoban TF. Sleep disorders in children. Ann N Y Acad Sci 2010;1184:1-14.

[49] Madrid-Valero JJ, Rubio-Aparicio M, Gregory AM, Sánchez-Meca J, Ordoñana JR. Twin studies of subjective sleep quality and sleep duration, and their behavioral correlates: systematic review and meta-analysis of heritability estimates. Neurosci Biobehav Rev 2020;109:78-89.

[50] Cooper H, Hedges LV, Valentine JC. The handbook of research synthesis and meta-analysis. Russell Sage Foundation; 2019.

[51] Sanchez-Meca J, Marin-Martinez F. Confidence intervals for the overall effect size in random-effects meta-analysis. Psychol Methods 2008;13(1): $31-48$.

[52] Hartung J, Knapp G. On tests of the overall treatment effect in meta-analysis with normally distributed responses. Stat Med 2001;20(12):1771-82. 
[53] Huedo-Medina TB, Sánchez-Meca J, Marín-Martínez F, Botella J. Assessing heterogeneity in meta-analysis: Q statistic or I2 index? Psychol Methods 2006;11(2):193-206.

[54] Duval S, Tweedie R. Trim and fill: a simple funnel-plot-based method of testing and adjusting for publication bias in meta-analysis. Biometrics 2000;56(2):455-63.

[55] Rothstein HR, Sutton AJ, Borenstein M. Publication bias in meta-analysis: prevention, assessment and adjustments. Willey; 2005.

[56] Egger M, Smith G, Schneider M, Minder C. Bias in meta-analysis detected by a simple, graphical test. BMJ Br Med J (Clin Res Ed) 1997;315(7109):629-34.

[57] Knapp G, Hartung J. Improved tests for a random effects meta-regression with a single covariate. Stat Med 2003;22(17):2693-710.

[58] Rubio-Aparicio M, Lopez-Lopez J, Viechtbauer W, Marin-Martinez F, Botella J, Sanchez-Meca J. Testing categorical moderators in mixed-effects meta-analysis in the presence of heteroscedasticity. J Exp Educ 2020;88(2):288-310.

[59] López-López JA, Marín-Martínez F, Sánchez-Meca J, Van den Noortgate W, Viechtbauer W. Estimation of the predictive power of the model in mixedeffects meta-regression: a simulation study. Br J Math Stat Psychol 2014;67(1):30-48.

[60] Viechtbauer W. Conducting meta-analyses in R with the metafor package. J Stat Software 2010;36(3):1-48.

[61] Plomin R, DeFries JC, Knopik VS, Neiderhiser JM. Top 10 replicated findings from behavioral genetics. Perspect Psychol Sci 2016;11(1):3-23.

[62] Mayhew AJ, Meyre D. Assessing the heritability of complex traits in humans: methodological challenges and opportunities. Curr Genom 2017;18(4): $332-40$.

[63] Manolio TA, Collins FS, Cox NJ, Goldstein DB, Hindorff LA, Hunter DJ, et al. Finding the missing heritability of complex diseases. Nature 2009;461(7265):747-53.
[64] Yang J, Zeng J, Goddard ME, Wray NR, Visscher PM. Concepts, estimation and interpretation of SNP-based heritability. Nat Genet 2017;49(9):1304-10.

[65] Lind MJ, Hawn SE, Sheerin CM, Aggen SH, Kirkpatrick RM, Kendler KS, et al. An examination of the etiologic overlap between the genetic and environmental influences on insomnia and common psychopathology. Depress Anxiety 2017;34(5):453-62.

[66] Gregory AM, Buysse DJ, Willis TA, Rijsdijk FV, Maughan B, Rowe R, et al Associations between sleep quality and anxiety and depression symptoms in a sample of young adult twins and siblings. J Psychosom Resvol. 71. England: Elsevier Inc; 2011. p. 250-5.

[67] Gasperi M, Herbert M, Schur E, Buchwald D, Afari N. Genetic and environmental influences on sleep, pain, and depression symptoms in a community sample of twins. Psychosom Med 2017;79(6):646-54.

[68] Song W, Torous J, Kossowsky J, Chen CY, Huang H, Wright A. Genome-wide association analysis of insomnia using data from Partners Biobank. Sci Rep 2020;10(1):6928.

[69] Grasby K, Jahanshad N, Painter J, Colodro-Conde L, Bralten J, Hibar D, et al. The genetic architecture of the human cerebral cortex. Science 2020;367(6484):1340 [+].

[70] Vgontzas AN, Fernandez-Mendoza J, Liao D, Bixler EO. Insomnia with objective short sleep duration: the most biologically severe phenotype of the disorder. Sleep Med Rev 2013;17(4):241-54.

[71] Lebowitz MS. The implications of genetic and other biological explanations for thinking about mental disorders. Hastings Cent Rep 2019;49(Suppl 1): S82-7.

[72] Lebowitz MS, Ahn WK. Effects of biological explanations for mental disorders on clinicians' empathy. Proc Natl Acad Sci U S A 2014;111(50): 17786-90. 